\title{
CONSUMER BEHAVIOR ANALYSIS BY GRAPH MINING TECHNIQUE
}

\author{
KATSUTOSHI YADA* \\ Faculty of Commerce, Kansai University \\ 3-3-35 Yamate, Suita, Osaka 564-8680, Japan \\ yada@ipcku.kansai-u.ac.jp \\ HIROSHI MOTODA ${ }^{\dagger}$ and TAKASHI WASHIO ${ }^{\ddagger}$ \\ ISIR, Osaka University \\ 8-1 Mihogaoka, Ibaraki \\ Osaka 567-0047, Japan \\ †motoda@ar.sanken.osaka-u.ac.jp \\ †washio@ar.sanken.osaka-u.ac.jp \\ ASUKA MIYAWAKI \\ Faculty of Commerce, Kansai University \\ 3-3-35 Yamate, Suita \\ Osaka 564-8680, Japan \\ da10645@edu.kansai-u.ac.jp
}

\begin{abstract}
In this paper, we discuss how graph mining system is applied to sales transaction data so as to understand consumer behavior. First, existing research of consumer behavior analysis for sequential purchase pattern is reviewed. Then we propose to represent the complicated customer purchase behavior by a directed graph retaining temporal information in a purchase sequence and apply a graph mining technique to analyze the frequent occurring patterns. In this paper, we demonstrate through the case of healthy cooking oil analysis how graph mining technology helps us understand complex purchase behavior.
\end{abstract}

Keywords: Graph mining; graph structure; consumer behavior; purchase pattern; cooking oil market.

\section{Introduction}

In Japan, "Health" related products have become a major focus of attention among consumers and industries in recent years. ${ }^{1}$ Because of the fact that health related products have a high-added value, it is understandable that food retailers who are groping for sales promotions that do not rely on price reduction to attract customers

\footnotetext{
* Corresponding author.
} 
find that the consumer group that purchases said health related products is a very attractive one indeed. However, these retailers who are attempting to lure ever greater numbers of high value customers at their shops by proposing effective food menus will find themselves faced with the difficult dilemma of trying to extract the characteristics of these consumers from their complex purchasing behavior. ${ }^{2-4}$

Simply analyzing the contents of these consumer's shopping baskets is not enough to shed light on their purchasing pattern and lifestyles. It is also necessary to draw out the characteristics of the relationships existing amongst groups of products and relationship amongst products when a multitude of products are bought at one time. ${ }^{5}$ In this paper, we present the application by using graph mining technique to understand chance, health food boom to analyze purchasing historical data from the view point of consumer behavior and to create the trigger to discuss and communicate future scenario.

Up to the present, we have developed a variety of analysis methods that can be used to analyze the purchase history data of consumers. For example, we developed E-BONSAI ${ }^{6,7}$ which makes use of sequence analysis technique as a way to carry out temporal sequential analysis of categorical data and were able to successfully extract the characteristics found in consumer purchasing patterns. However, although it is possible to analyze the sequential patterns of limited product groups for which E-BONSAI is designed, it is not possible to resolve the above-mentioned problems by sequential pattern analysis. It became necessary for us to extract the characteristics from the products purchased in multiple purchase patterns along with their purchasing sequence.

In our research, we make proposals using characteristic patterns extracted from temporal sequences of purchased product groups that are represented as graph structured data. Graph structure is effective and useful to express complicated forms of data and phenomena. There have already been several algorithms for mining graphs and they are utilized to analyze chemical compounds and medical data. We believe that by applying these graph mining technique to the marketing field it will be possible to discover new implication that were not possible to be detected by the traditional forms of technology.

In this paper, we apply graph mining technique to the FSP data of supermarkets in an attempt to discover new opportunities through interactions of retailers and experts employed by a variety of manufacturers. We will extract characteristics of consumer behavior in salad oil market of Japan and present the possibility to apply graph mining to business fields.

\section{Analyzing the Behavior of Consumers Using Graph Mining Technology}

Graph mining is a technique used to extract characteristic patterns from a variety of graph structured data. ${ }^{8}$ The graph structure is a nice way of representing and 
explaining complex data forms and phenomena but because of its strong expressiveness, its computational complexity has been a problem to extract specific patterns. However, recent development has made it possible to perform a complete search in extracting all the subgraph in a reasonable computation time. AGM algorithm ${ }^{9}$ is one of the most advanced algorithms for graph mining and is able to deal with directed/undirected and colored/uncolored graphs. While graph mining research is still in the developmental stage, there is a fair amount of research being carried out already concerning its practical applicability.

For example, graph mining is applied to extract patterns from chemical compound data. In chemical domain molecular structure of chemical substances has always been expressed using graph structures, and thus it is natural that molecular analysis is one of the most frequently challenged application area of graph mining. In fact, graph mining technique successfully extracted meaningful substructures that cause carcinogenicity in organic chlorine compounds.

Although various types of work are currently being carried using graph mining, to the best of our knowledge, we are the first to apply graph mining technique to the business and marketing field. We have applied graph mining technique to POS data, which includes customer ID that has been accumulated in the retailing industry, in order to investigate the possibilities of applying graph mining to the marketing research field.

\subsection{Graph structures and consumer behavior}

Based on the POS data in Table 1 that include customer ID information, we have tried to express the purchase behavior of consumers using graph structure. The data shown in Table 1 is a detailed sales record of a single consumer following the purchase of salad oil. After purchasing salad oil, the customer ID:1 visited the store 2 times and purchased multiple products from different categories.

Using a graph makes it possible to express in extreme detail purchasing information concerning the composition of products as to when and where multiple products were purchased at one time. Figure 1 shows the purchasing behavior of the customer ID:1 using a graph structure. The root of the graph shows the purchase of "Healthy Okona" salad oil. On the following visit to the store (6-days later) the consumer

Table 1. Example of POS data including ID information.

\begin{tabular}{ccccc}
\hline ID & Date & Category & Goods & Price zone \\
\hline 1 & 2004 Feb02 & Beer & Super D & High \\
1 & 2004 Feb02 & Egg & Organic & High \\
1 & 2004 Feb02 & Milk & Unmei Milk & High \\
1 & 2004 Feb02 & Mayo & Mayo Q & Normal \\
1 & 2004 Feb12 & Egg & Organic & High \\
1 & 2004 Feb12 & Bread & Genmai bread & High \\
1 & 2004 Feb12 & Milk & Honeboso Milk & Low \\
\hline
\end{tabular}




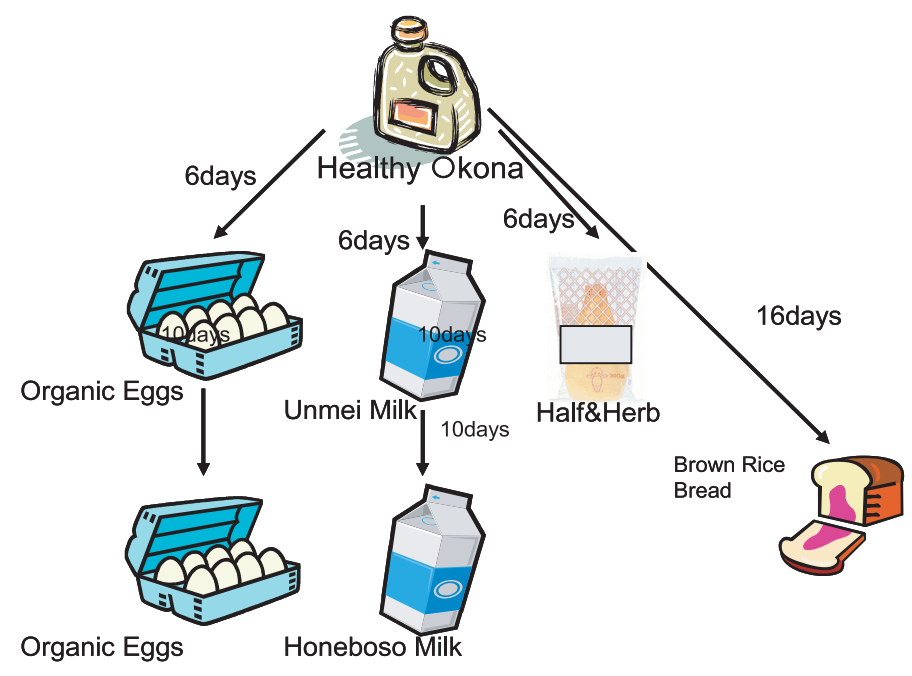

Fig. 1. Graph structured data and purchasing behavior.

purchased "Organic eggs," "Unmei milk" and "Half \& Herb." The arrows connecting these items represent the purchasing sequence. Next to the edges linking the respective products, labels are attached that indicate the intervals between these purchases. The "Organic eggs" and "Honeboso milk" which were purchased on the following visit to the store are connected by arrows with the products purchased in the same category on the previous visit, and these also have labels attached which indicate the number of days that have elapsed since the previous purchase date. Also, the categories for products such as "Brown rice bread" which were not purchased on the preceding visit are linked to the salad oil.

The data contained in this graph includes not only the information about simultaneous purchases on the product level but also time-sequence information about multiple purchases and information on groups of purchased products, making it possible to extract characteristic patterns from this information which can lead to the discovery of new knowledge which were unobtainable by traditional methods.

\subsection{Types of graph structure in consumer purchase behavior}

There are various methods of expressing consumer purchase behavior in graph structures. In this paper, I will introduce two types of graph structure, which take into consideration the analysis objectives and computation time; namely the tree graph and the star graph.

\subsubsection{Tree graphs}

Tree graphs are a graph structure in which links are made between the root node and each of the other nodes. Figure 2(a) is the free-fall-type tree graph, the most typical 


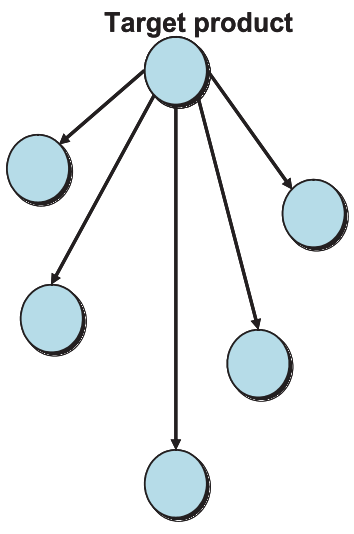

(a)

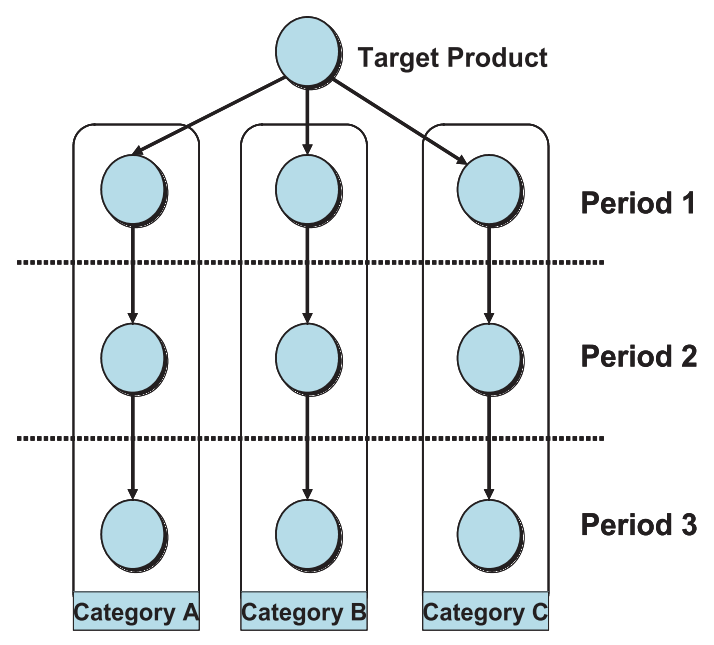

(b)

Fig. 2. Free-fall-type and matrix-type tree graphs.

type. Usually, the root node is the target product, and is the subject of analysis; and products which are purchased later and labels indicating the interval between purchases are grafted using links. However, if the period of analysis extends over a long period of time, or if many products are purchased all at once at a supermarket, etc, then this type of graph becomes too complicated, and characteristic subgraphs and rules extracted is not easily understood and interpreted.

Consequently, we have adopted the matrix-type tree graph structure as shown in Figure 2(b). By having the user select their desired product category types which are related to the target product, and then by having the number of analysis periods restricted to just a few, we can efficiently extract pattern characteristics. It is possible to attach interval periods in terms of days to the link, and product names, price brackets, etc to each of the nodes. We have used this matrix-type tree graph in the analysis of consumer behavior in the salad oil market below.

\subsubsection{Star graphs}

Star graphs (Fig. 3) express a consumer's purchases on a single outing in the shape of a star, with the core node being the single purchase point of time, the satellite nodes displaying the purchased products, and having labels of product names or price brackets attached. Next, first-time purchases and second-time purchases are joined by a link with a label attached, indicating the number of days between purchases. This can only be repeated for the number of times the customer, who is the subject of analysis, makes purchases.

These two types of graph structures are chosen to suit a purpose of the analysis. Tree graphs are mostly used for a particular product, to extract related product groups or purchase patterns, in order to forecast the effectiveness of sales 


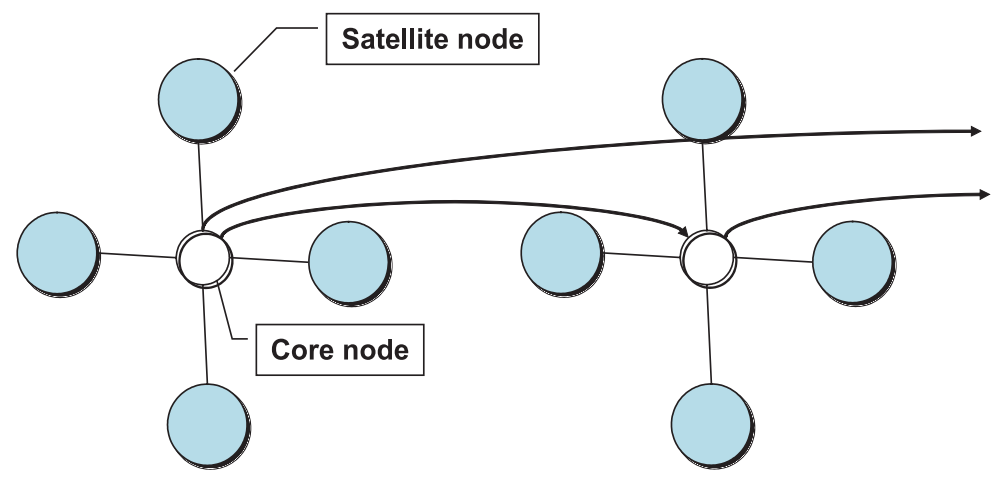

Fig. 3. Star graph.

promotions, discover products related to new products, etc. Star graphs are used for particular customers to extract their purchased products or purchase pattern characteristics. Compared to other graphs, data loss is minimal, and they are best suited to the analysis of complex consumer behavior. However, computations to extract these powerful forms of expression are not insignificant. Consequently, contrivances are necessary, such as restricting related products to those in which the user is interested.

In the next chapter, we shall analyze customers of the salad oil market and discover valuable knowledge by using data from these types of graph structures which express consumer purchase behavior.

\section{Analyzing the Behavior of Consumers in the Salad Oil Market}

\subsection{Salad oil market}

The keyword, "Health" is one of the most powerful consumer and industrial oriented attention-getters in the Japanese food and food-related markets. In the midst of an on-going deflationary economy, products marketed under the keyword of "Health" are bought at high prices and it is believed that growth in this sector of the market will continue to expand for the time being. The item that played a large role in spurring on this growth was a salad oil product that was marketed under the image of "Health" by a manufacturer. Although this product was a newcomer to the salad oil market, it has occupied over a $10 \%$ share of the market, and we are continuing to see a constant influx of products that play up the "Health" aspect from almost all concerned manufacturers making this an important segment of the overall market. In this paper, we refer to this group of products that are marketed under the keyword of "Health" as health-oriented salad oils.

The salad oil market is composed of 17 health-oriented salad oil products and 16 normal salad oil types and the market share of health-oriented salad oil products in the overall salad oil market is over $30 \%$. While there are obviously some products with strong brandname appeal there is a big switchover taking place basically to 
these health-oriented salad oil products and we feel that it is appropriate to consider the users of these products as making up a single consumer segment. Fundamental analysis reveals that when compared with consumers who purchase other types of more ordinary salad oils, users of this segment possess several special characteristics including a tendency to use oil itself in smaller quantities than their counterparts and other types of imbalances were also seen in the other products they purchased as well.

We also discovered that among the segment of consumers who purchase healthoriented salad oil products there exists a consumer segment that only purchase these health-oriented salad oil products and one that purchases these products in conjunction with other products. In this paper, we will refer to the former group as "Healthy users" and the latter group as "Dual users." In general "Healthy users" purchase salad oils at high price while "Dual users" show an extremely high level of response to salad oils that are on sale. However, when compared with normal users, both of these segments tend to purchase products at high prices excluding salad oil products and the ratio of the purchase of products on sale is low. Hereafter, we focus our attention of analysis on these three consumer segments consisting of "Healthy users," "Dual users" and "Normal users."

\subsection{Preprocessing and transformation of data}

We made use of FSP data of the Kanto area's GMS (General Merchandizing Store) covering a 1-year period running from July 2002 to June 2003. During this period salad oil purchases were higher than the average with 2979 "Healthy users" (Comprising more than $66 \%$ of the category's total) purchasing mainly health-oriented salad oil products, 3437 "Dual users" (Comprising less than 66\% of the healthoriented salad oil total) purchasing health-oriented salad oil products and other products, and 12,088 "Normal users" not purchasing any health-oriented salad oil products. We carried out analysis to discover the characteristics of the purchasing behaviors of "Healthy users" and "Dual users" in the 1-month period following their health-oriented salad oil product purchases. From the principal food, product categories made up of 50-product groups where we extracted data for eggs, milk, bread and mayonnaise because of their strong relationships with salad oils. We divided the price zones of the market prices of each individual product associated with these product groups into the ranks of a high price zone, a middle price zone and a low price zone. We also broke the 1-month analysis period into 310 -day periods and analyzed the purchased products groups within the aforementioned periods.

\subsection{Special characteristics of the extracted consumer behavior patterns}

When we analyzed the above-mentioned data using graph mining technique we discovered several characteristic aspects of purchasing behavior regarding product purchasing and price zones. 


\subsubsection{Tendencies of "Healthy users" to purchase high price zone products}

In each of the 3 periods "Healthy users" simultaneously purchased high price zone products spanning over different categories at a ratio of around 10 to $15 \%$ which was relatively higher than the $5 \%$ found with "Dual users". Further, when compared with "Dual users" it was discovered that many more "Healthy users" tended to make consecutive purchases of high price zone products in the categories of bread, eggs and milk as shown in Fig. 4 . The $10.8 \%$ ratio of "Healthy user" consumers who purchased high price zone products 6 times or more from among the 3 categories in the 3 periods greatly exceeded the $4.7 \%$ ratio found with "Dual users."

"Healthy users" did not only show a tendency to purchase products that have a low frequency of purchase such as salad oils in the high price zone, but also tended to purchase items with a high frequency of purchase such as bread, eggs or milk in the high price zone. This makes the "Healthy user" segment an extremely attractive one to retailers and manufacturers. In our meetings with specialists a proposal to create a new consumer segment based on these product groups was suggested and we have since launched a project to do so.

\subsubsection{Importance of detailing these categories}

We discovered that both types of users exhibited a pattern of continuing to purchase products in the high price zone categories in which they made purchases in the first

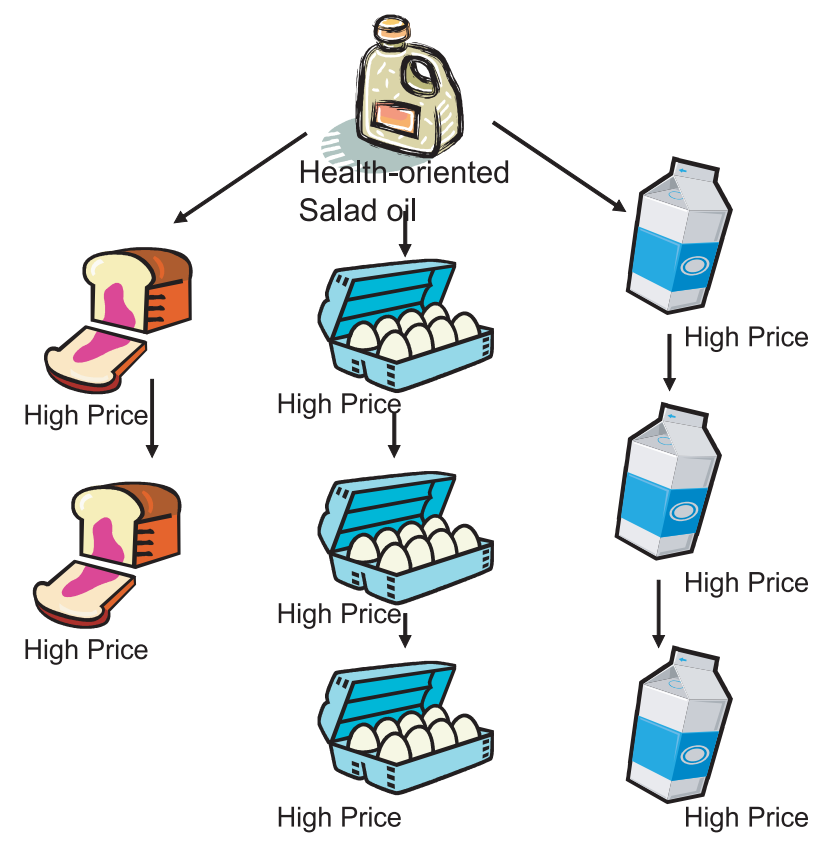

Fig. 4. Tendencies of healthy users. 
10-days following the purchase of a health-oriented salad oil product. In the previous section, we came across a rule which showed that "Healthy users" possessed a strong tendency to purchase products in the high price zone in all categories, and we also verified that "Dual users" also possess tendencies to make purchases in particular high price zones and that they tend to continue to make these purchases.

This tendency of "Dual users" does not stem from the purchase of a healthoriented salad oil product but is rather assumed to be more like a consumer who originally possesses a loyalty to a particular category making trial purchase of a health-oriented salad oil product that has been marketed at a sale price. In particular, a large ratio of "Dual users" continues to purchase milk in the high price zone and this can be said to be true of "Healthy users" as well. The purchasing frequency of salad oil is lower than that of milk or eggs and thus it may be that consumers who purchase these products often may also tend not to pay too much attention to salad oil products.

\section{Conclusion}

In this paper, we carried out an initial attempt that involved applying graph mining to the behavior analysis of consumers in the marketing field, converting the purchase history of real world industrial data into graph structures. Using graph structured data to represent consumer behavior makes it possible to effectively convey information that possesses temporal sequence property and in particular relationships existing between multiple product groups purchased in multiple purchase settings. By extracting patterns discovered by graph mining in analyzing consumer behavior in the salad oil market, we were able to define several characteristic patterns. Graph mining has so far not been applied to analyze the behavior of consumers. Our results are encouraging and we hope that this would be a valuable initial step toward a new type of consumer behavior analysis to understand a chance in the purchasing historical data. The future directions of our work are to present scenario communication process among participants after understanding the specific events by using graph mining application and to evaluate the performance of business action emerged from these various processes.

\section{Acknowledgments}

Research of this paper is partly supported by the Grant-in-Aid for Scientific Research on Priority Areas "Active Mining" by the Ministry of Education, Science, Sports and Culture of Japan.

\section{References}

1. K. Yada, H. Motoda, T. Washio and A. Miyawaki, Consumer behavior analysis by graph mining technique, in Proc. of KES 2004, Lecture Notes in Artificial Intelligence, Vol. 3214 (Springer-Verlag, Berlin Heidelberg, 2004), pp. 800-806. 
2. Y. Hamuro, N. Katoh, N. Matsuda and K. Yada, Mining pharmacy data helps to make profits, Data Mining and Knowledge Discovery 2(4) (Springer-Verlag, New York, 1998), pp. 391-398.

3. K. Yada, The future direction of active mining in the business world, Frontiers in Artifi-cial Intelligence and Applications 79 (IOS Press, Amsterdam, 2002), pp. 239-245.

4. K. Yada, Knowledge discovery process and introduction of domain knowledge, in Innovations of Knowledge Management, ed. B. Montano (IRM Press, New York, 2004), pp. 86-98.

5. K. Fujisawa, Y. Hamuro, N. Katoh, T. Tokuyama and K. Yada, Approximation of optimal two-Dimensional association rules for categorical attributes using semidefinite programming, in Proc. First Int. Conf. DS'99, Lecture Notes in Artificial Intelligence, Vol. 1721 (Springer-Verlag, New York, 1999), pp. 148-159.

6. Y. Hamuro, H. Kawata, N. Katoh and K. Yada, A machine learning algorithm for analyzing string patterns helps to discover simple and interpretable business rules from purchase history, Progress in Discovery Science, Lecture Notes in Artificial Intelligence, Vol. 2281 (Springer-Verlag, Berlin Heidelberg, 2002), pp. 565-575.

7. Y. Hamuro, N. Katoh, E. H. Ip, S. L. Cheung and K. Yada, Combining information fusion with string pattern analysis: A new method for predicting future purchase behavior, in Information Fusion in Data Mining, Studies in Fuzziness and Soft Computing, ed. V. Torra, Vol. 123 (Springer-Verlag, Berlin Heidelberg, 2003), pp. 161-187.

8. A. Inokuchi, T. Washio, Y. Nishimura and H. Motoda, General framework for mining frequent structures in graphs, in Proc. Int. Workshop on Active Mining (SpringerVerlag, Berlin Heidelberg, 2002), pp. 23-30.

9. A. Inokuchi, T. Washio and H. Motoda, An apriori-based algorithm for mining frequent substructures from graph data, in Proc. 4th European Conf. Principles and Practice of Knowledge Discovery in Databases (Springer-Verlag, Berlin Heidelberg, 2000), pp. 13-23. 